\title{
Simulation of Peak Ground Acceleration by Artificial Neural Network and Radial Basis Function Network
}

\author{
Ali Nasrollahnejad \\ Master of Science, Invited lecturer of pay am Nour University of gorgan, Iran. P.O. Box 49175-876, Tel-Fax No: 0171- \\ 4436236, Iran. (alinasrollah64@gmail.com) \\ Hamid rajabi, Mohammad Bagher Nasrollahnejad, Samaneh Tahmasebi \\ Master of Science, Power Engineering, University of Aliabad, Iran \\ PHD student of Electronic Engineering, University of semnan, Iran \\ PHD student of Mathematic, University of Shahroud, Iran \\ Email: \{hamid-rajabi0171@yahoo.com,nasrollahnejad@gmail.com, Samaneh.tahmasebi.63@gmail.com\}
}

\begin{abstract}
Recording of ground motions with high amplitudes of acceleration and velocity play a key role for designing engineering projects. Here we try to represent a reasonable prediction of peak ground acceleration which may create more than g acceleration in different regions. In this study, applying different structures of Neural Networks (NN) and using four key parameters, moment magnitude, rupture distance, site class, and style of faulting which an earthquake may cause serious effects on a site. We introduced a radial basis function network (RBF) with mean error of 0.014 , as the best network for estimating the occurrence probability of an earthquake with large value of PGA $\geq 1 \mathrm{~g}$ in a region. Also the results of applying back propagation in feed forward neural network (FFBP) show a good coincidence with designed $\mathrm{RBF}$ results for predicting high value of PGA, with Mean error of 0.017 .
\end{abstract}

Index Terms-Peak ground acceleration, Moment magnitude, Rupture distance, Site class, Radial basis function, General regression neural network.

\section{INTRODUCTION}

Reviewing physical factors which influence ground motions is done by several authors and they are often applied in estimating ground motions [1-3]. These group interpretations also are differentiated between source factors and site. Also, source parameters mentioned include balance of ground motions that contains specified factors of total amount of the energy released by resource like source intensity and specified parameter of rate of releasing energy. Also ground motion near fountain are influenced more powerfully by phenomenon close to fountain, therefore, works of near fountain like jump and guidance have special points [4-5].

Peak ground acceleration is one of the most important parameters which is often investigated in the studies related to earthquakes damages. In [1] mostly estimated by reduction relations and is expanded by analyzing data regression of powerful motions. In [1] is investigated the way of applying neural networks in predicting peak ground acceleration using data of powerful motions in Turkey. In regression approach, PGA is repeatedly calculated as function of parameters of (earthquake magnitude, distance from source to system, general condition of system [6].

Comparing the correlation coefficient gained with neural network and regression, it can be concluded that neural network has higher level of coefficient. Also models of neural networks have weaker structures in the ability of generalization and always perceive effects of input parameters in predicting PGA as (1) and (2) [7].

$$
\begin{gathered}
R^{n}\left(M, M^{n}\right)=\frac{\operatorname{Cov}\left(M, M^{n}\right)}{\sqrt{\operatorname{Cov}(M, M) \operatorname{Cov}\left(M^{n}, M^{n}\right)}} \\
r=\frac{\sum_{i=1}^{m}\left(x_{i}-\bar{x}\right)\left(y_{i}-\bar{y}\right)}{\sqrt{\sum_{i=1}^{m}\left(x_{i}-\bar{x}\right)^{2}} \sqrt{\sum_{i=1}^{m}\left(y_{i}-\bar{y}\right)^{2}}}
\end{gathered}
$$

In (1), covariance matrix of magnitude of earthquake moment was used for sensors 2, 3 and 4. Also estimating moment indicates a clear increase in time and number of censors. $\mathrm{n}$ also is number of timesteps related to sensor number 1 .

In (2), $\mathrm{X}_{\mathrm{i}}$ is records and $\overline{\mathrm{X}}$ is mean level. $\mathrm{Y}_{\mathrm{i}}$ is output estimated and $\overline{\mathrm{y}}$ is the mean.

In this paper System Methods and calculations related to gathering data and preparing for examining and testing neural networks is presented in section II. Three neural networks are introduced and investigated in section III. Finally the results concluded in section IV. 


\section{System Methods And CALCUlations}

One of the problems noticed by seismologists is occurring earthquakes in which peak acceleration of ground motion is unexpectedly more than $1 \mathrm{~g}$.

Ref [8] used valuable data in such earthquakes and investigated physical process caused by them. PGA parameter is often estimated by reducing relations and analyzing regression.

The first set of acceleration recorders investigated in the study of [8] contain 44 horizontal components and 22 vertical ones in records of cases in which peak acceleration larger than $1 \mathrm{~g}$ are estimated and correspond with 43 records gained from 40 stations during 26 earthquakes.

In the first phase of neural network model, weight parameters are multiplied by their own corresponding input data and their scales are based on level of importance. After combining input of changing scale given with each other, the signal gained is lead to section in which transfer function is imposed on the signal.

The output of this part is considered as a true output of neural network. Level of weights' coefficient and modifying them during training are regulated along with learning principles. Learning principles can be classified into two parts of supervised and unsupervised.

In this research, 38records are considered for training the network and 8 records are considered for testing the network and 14records for training the network. Also a common network is created and from among several types of general feed forward network, Radial basis function and general regression we chose one of them. Also networks parameters is chosen which contains layers, number of neurons, rate of momentum, rate of learning, transfer function and maximum of repetitions and after that input display is specified. Then various networks are dealt with and regression charts, error square mean and momentum are investigated for each network as well. Finally, the least error mean is chosen among various networks and it will be considered as the best network. Equation (3) is used for normalization of 60 available records.

The results of output and the error gained from three neural networks are considered in the results section.

$$
\mathrm{N}=\left(\mathrm{X}-\mathrm{X}_{\min }\right) /\left(\mathrm{X}_{\max }-\mathrm{X}_{\min }\right) \times 0.8+0.1
$$

\section{DISCUSSION}

Three neural networks are introduced and investigated in this section [9-10].

\section{A. Feed forward Back Propagation neural Network}

A general sahpe of multi-layer perceptron network has one or several more connections to other layers. Theoretically it can demonstrate every problem which can be proved by general feed forward network [9-10]. Also by testing, general feed forward network can solve the problem more accurately.
For example, we have spiral issues and it is efficient to say that preceptor network needs one hundred times more training repetition to general feed forward network with the same number of processing elements.

This network usually uses training algorithms of statistical error propagation, Lundberg Marcoart and descending gradient. Function of transferring input layers are chosen by Log sigmoid, middle layers are chosen by Tangent and last layers are chosen by linear ones that as shown as in Fig.1 and Table1.

Table 1. Level of mean square error, mean normalized error, mean absolute error, minimum absolute error, maximum absolute error and correlation coefficient calculated for general feed forward network by analyzer (neuro solution) neural network.

\begin{tabular}{|c|c|}
\hline Performance & E \\
\hline MSE & 0.125191843 \\
\hline NMSE & 0.854933892 \\
\hline MAE & 0.253670009 \\
\hline Min Abs Error & 0.012040995 \\
\hline Max Abs Error & 0.829856873 \\
\hline R & 0.415382216 \\
\hline
\end{tabular}

In this approach number of epocks is considered 1000 and level of input processing element is 10, learning training function is considered momentum and its value is 0.7 and transfer function is considered Tangoing axon. 38 records are considered for training network, 14 records for testing network and 8 records are considered for checking network.

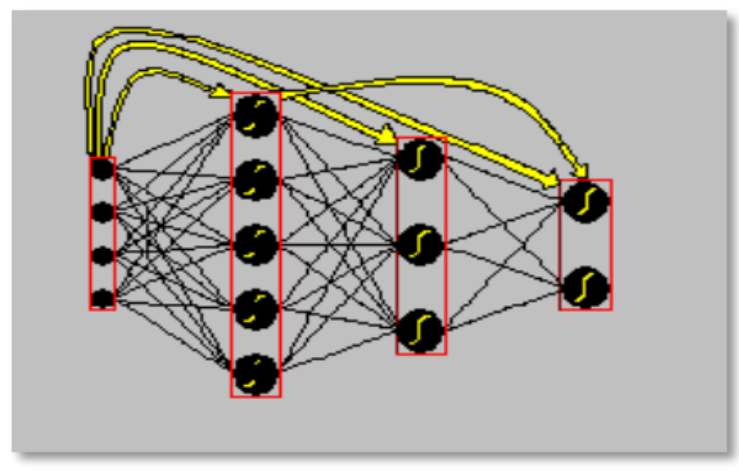

Fig 1. Two layered feedforward network designed.

Table 2. Sensivity factor was gained for input parameters.

\begin{tabular}{|cc|}
\hline Sensitivity & $e$ \\
\hline a & 0.161595205 \\
b & 0.051523174 \\
c & 0.260038734 \\
d & 0.072665706 \\
\hline
\end{tabular}




\section{B. General Regression Neural Network}

This network was also invented by donald espect for modelling and recognizing the system and it can be considered as a generalization from probable neural network. Probable network is used particularly for classifying models, while general regression network has wider applications as shown in Fig. 3 and Table I.

The first hidden layer which contains 5 neurons and the second hidden layer which included 3 neurons are preceded.

Function of transferring second and third layers are chosen by symmetrical Tanjong sigmoid and the last layer is usually considered linear and pure linear (Fig. 1). Then the data and rules between all neurons are processed and they are not for special neurons. The connection between neurons has no direction and uses training rules of Lundberg Marco art, descending gradient and feed forward back propagation such as other neural networks in order to train data of the network.

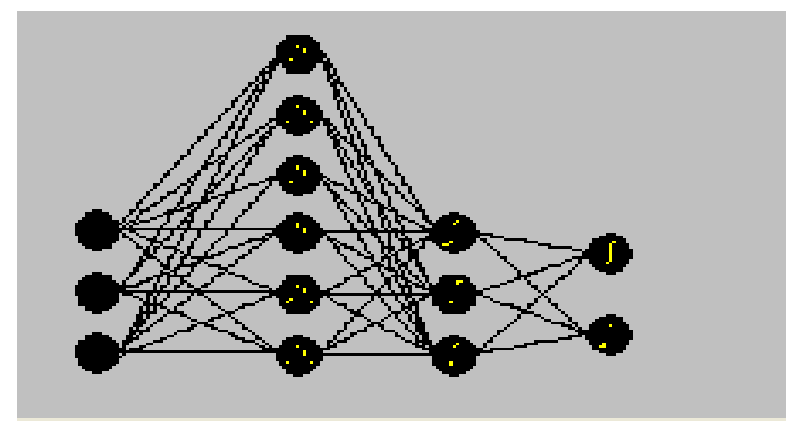

Fig 2. General regression neural network designed.

It can be observed (Fig. 2) that here we have four-layer network with two hidden layers. The first hidden layer has 6 neurons and the second one has 3 and that neurons do calculation operations on input data and act as a processor.

This network is a memory based network that provides estimates of continuous variables and converges to the underlying linear or nonlinear regression surface.

This general regression neural network is a one pass learning algorithm with a highly parallel structure. Even with sparse data in a multidimensional measurement space, the algorithm provides smooth transitions from one observed value to another. The algorithmic form can be used for any regression problem in which an assumption of linearity is not justified. The parallel network form should find use in applications such as learning the dynamics of a plant model for prediction or control [11].

It is a four-layer network (Fig. 2) with two middle (hidden) layers which the first hidden layer has 6 neurons and the second one has 3 neurons and processing of data is done between them by the neurons.
The first layer is in form of three-element column border and the last layer is in form of two-element column border. The last functions of transferring middle layer are usually chosen by log sigmoid.

\section{Radial basis neural network $(R B F)$}

Having verified applications, the network is one of the most desired neural networks and probably the real opponent of multi-layer perceptron. They are mostly inspired by traditional statistical model and these approaches were manifested in neural networks and are used widely as Figs. 4- 6.

As shown as in Fig.6 the training correlation coefficient is more that other ones and indicates that the network is trained well.

As shown as in Fig. 5, the results of testting and the network were good and desired network is stimulated.

Real architecture of RBF is a three-layer network. Input layer is a layer with splitting output and does not do any processing.

The second layer or hidden layer does a nonlinear mapping from input space with a higher dimension in which patterns become separable in linear form, therefore, the final layers does a simple total weight with a linear output.

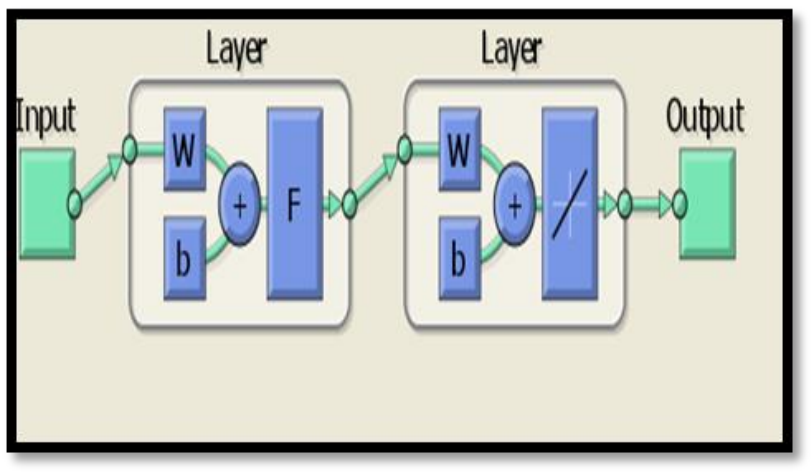

Fig 5. Radius basis function network designed.

The input layer is made up of source nodes that connect the network to its environment. The second layer applies a nonlinear transformation from the input space to the hidden space, in most applications the hidden space is of high dimensionality. The output layer is linear. supplying the response of the network to the activation pattern (signal) applied to the input layer [12].

The training, testing and validating correlation coefficient of radios basis function network are showed in Fig. 6.

The training, testing and validating mean square error of radios basis function network are illustrated in Fig. 7, The input to each RBF neuron is treated as a measure of the difference between data and a centre, which is a parameter of transfer function. 


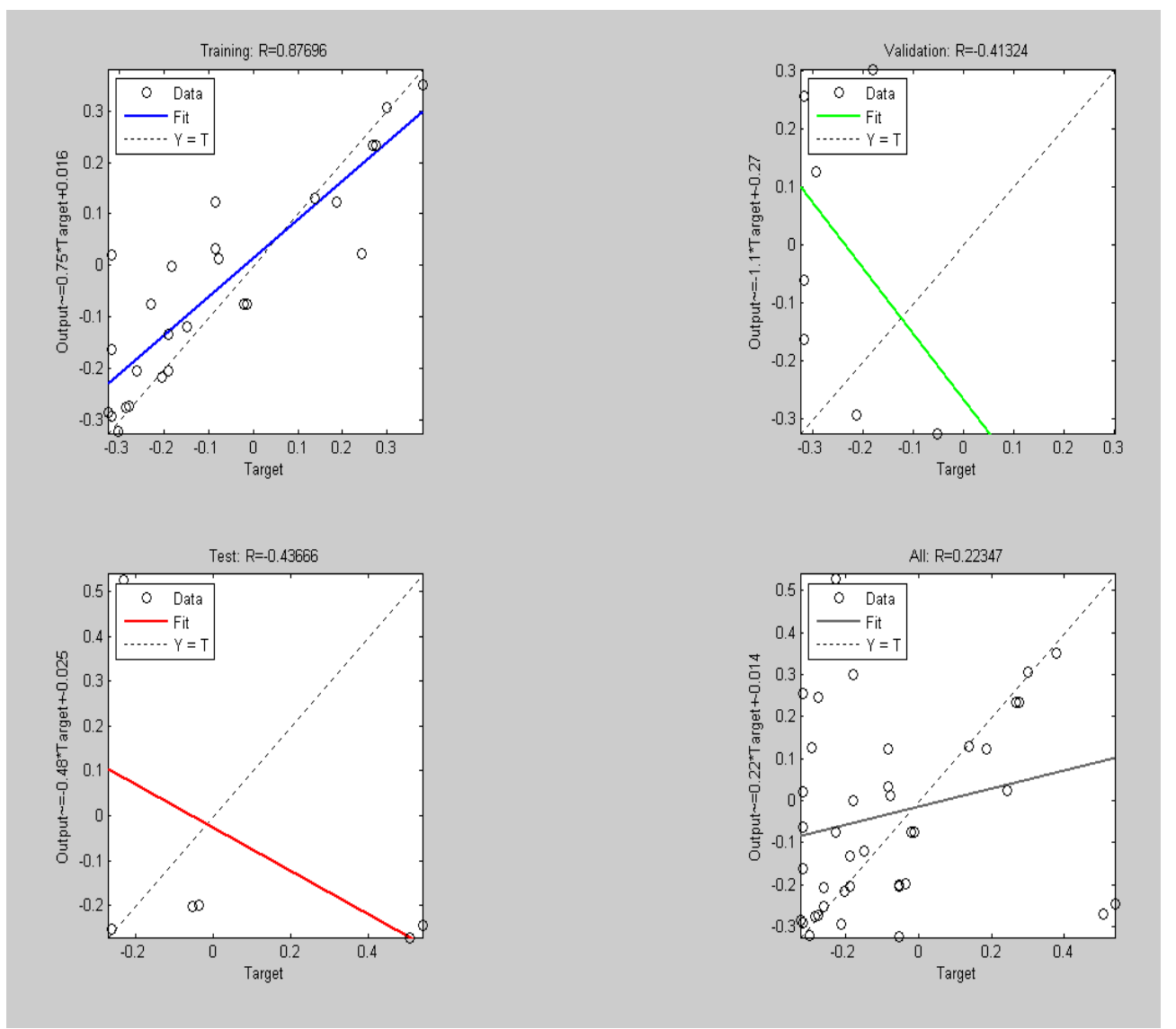

Fig 3. Correlation coefficient stimulated for training steps, testing and examining general regression neural network by Neural Network tool.

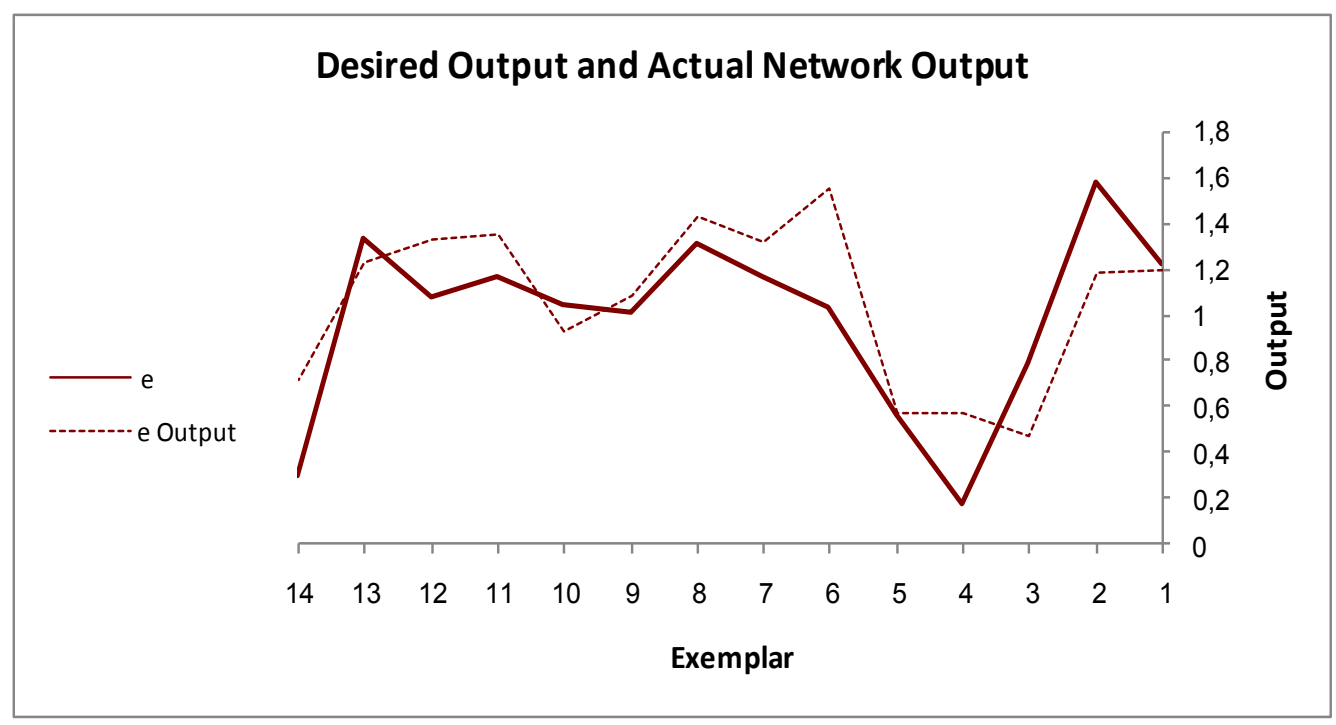

Fig 4. Comparing real output with output of radios basis function network test designed.

The transfer function of the neuron indicates the influence of data points at the centre. Generally this function is Gaussian and its centers can be chosen either randomly from the training data or they are iteratively trained or derived using techniques like K-means , Maxmin algorithms, Kohonen self organizing maps [13-15].

The output $y$ of an RBF is computed by the equation as follows:

$$
y=f(x)=\sum_{i=1}^{m} w_{i} . G\left\|x-c_{i}\right\|+w_{0}
$$

Where $\mathrm{m}$ is number of hidden nodes, $\mathrm{w}_{\mathrm{i}}$ is connections weights between the hidden neuron and output neuron, $\mathrm{x}$ is input vector, $\mathrm{c}$ is centre of the respective field. 

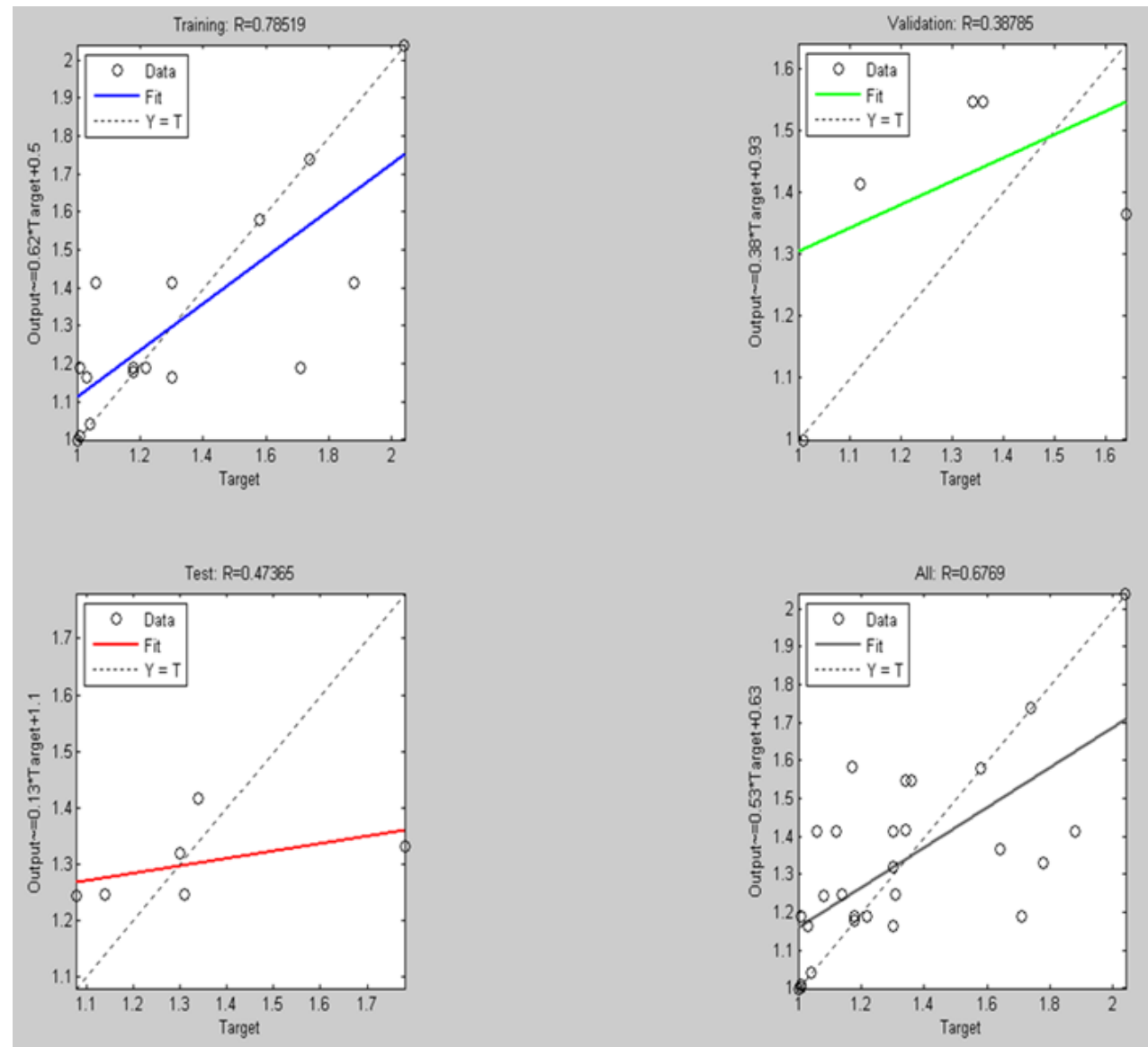

Figure 6. Correlation coefficient of training, testing and validating for Radius basis function Network has shown.

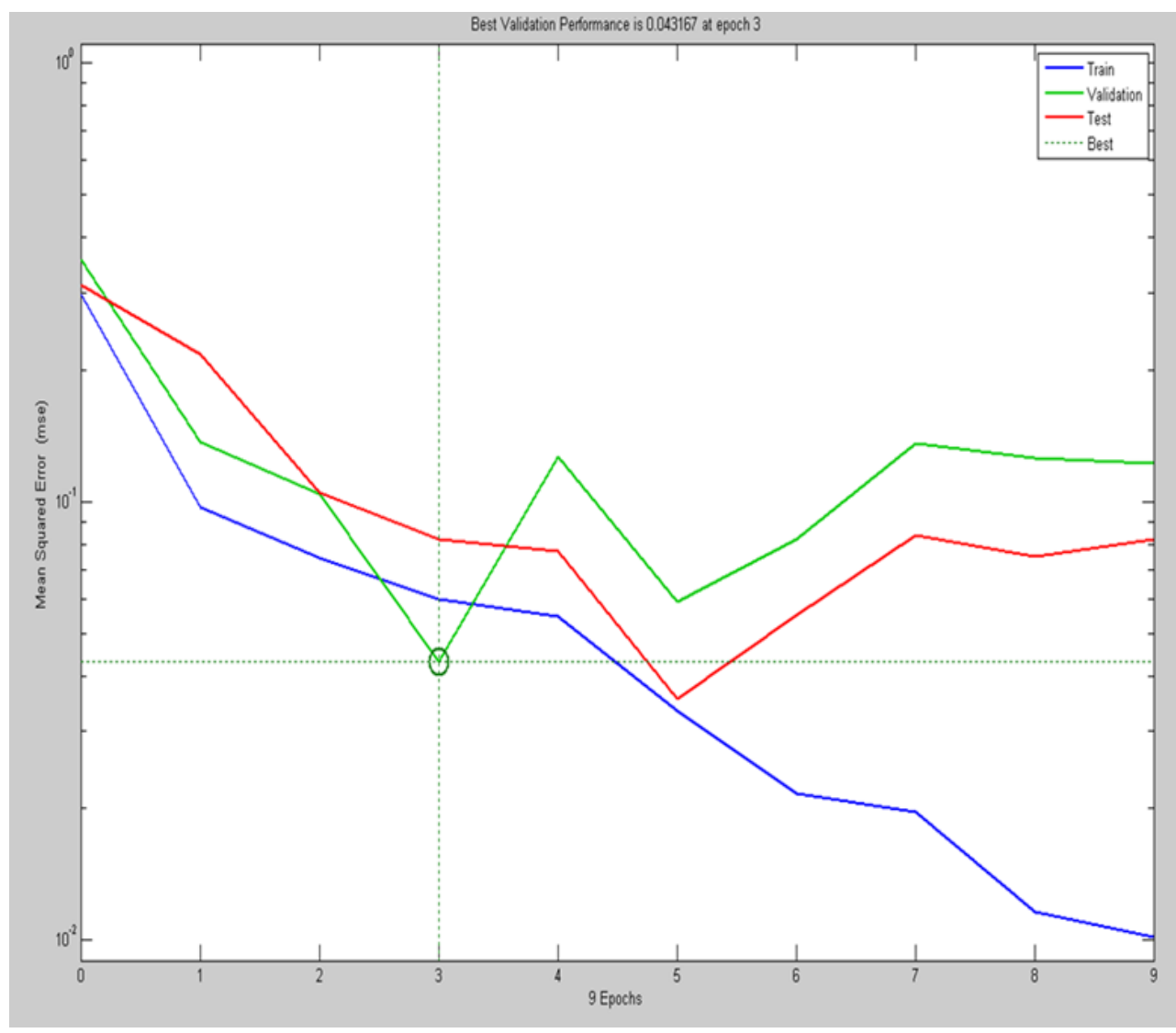

Fig 7. Best validation performance of Radius basis function Network has been calculated 0.043167 at epoch 3 . 


\section{CONCLUSION}

To predict peak acceleration of powerful motion of the earth across different areas in the world, training data was used with the networks mentioned. The highest level of correlation coefficient for radius basis function network is equal with 0.67 and the least MSE is related to radius basis function network with error 0.014. The most MSE error is for general regression network with error of 0.071 and in the end output of testing the network for Fernando seismograph is equal to 1.24 which fits well with real output of 1.22 .

\section{REFERENCES}

[1] Strasser .F.O., Bommer .J.J., Abrahamson NA., 2008, Truncation of the distribution of ground-motion residuals., Journal of Seismology ,12(1): 79-105.

[2] Moradzade .A, Bakhshi .E, 2006. Simulating photoelectric vision of oil building using artificial neural network,Journal of earth and space physic, vol.32,num.3,1-20,.

[3] Gullu.H ,Ergun.E , 2007, A neural network approach for attenuation relationships, Engineering Geology , 93,65-81.

[4] Huang. z.,and Liu .J,Wiliamson.M, and katsube.J , 1996 ,permeability prediction with artificial neural network modeling in the venture gaz field ,offshore eastern Canada Geophysic ,61,422-436.

[5] Shing .J, Jang. R, 1993, ANFIS:Adaptive Network Based Fuzzy Inference Systems,IEEE Transactions on Systems,vol.23,Num.3,MAY/JUNE,1993.

[6] Douglas. J ,2003, Earthquake ground motion estimation using strong motion records,.a review of equations for the estimation of peak ground acceleration and response spectral ordinates,.Earth-Sci,.Rev.61(1-2),43-104.

[7] Kreh.T, Chau. D , 2002, Neural network approach and microtremor measurements in estimating peak ground acceleration due to strong motion.,Engineering Software ,33,733-742.

[8] Strasser .F.O.,Bommer .J.J.,2009,Soil Dynamics and Earthquake Engineering , Large-amplitude ground-motion recordings and their interpretations,29,1305-1329.

[9] MaysamHedayati, Seyed Hossein Kamali, Reza Shakerian," Comparison and Evaluation of Intelligence Methods for Distance Education Platform", International Journal of Modern Education and Computer Science, Vol.4, No.4, May 2012.

[10] RichaShukla, ReenuShukla, AnupamShukla, Sanjeev Sharma, Nirupama Tiwari," Gender Identification in Human Gait Using Neural Network", International Journal of Modern Education and Computer Science, Vol.4, No.11, December 2012.

[11] Specht .F.D, 1991, A general regression neural network, Brief papers, $568-575$,

[12] Gunanaydin .K, Gunaydin. A, 2008, Peak ground acceleration prediction by artificial neural networks for northwestern turkey, 12 October 2008,

[13] S.Haykin, 1999, Neural Networks: A comprehensive foundation, Prentice-Hall, Englewood Cliffs, NJ, USA, 2nd edition.

[14] Kerth, T., Huang, C. \& Gunaratman, D. 2011. Neural network approach for analyzing seismic data to identify potentially hazardous bridges. Mathematical Problems in Engineering, 2011.
[15] De Lautour, O. R. \& Omenzetter, P. 2009. Prediction of seismic-induced structural damage using artificial neural networks. Engineering Structures, 31, 600-606.

\section{Authors' Profiles}

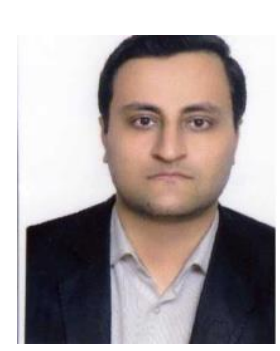

Ali nasrollahnejad was born in Gorgan, Iran. He received the M.Sc. degree in geophysics from ferdowsi University of mashhad, Iran. His main fields of interests are Earthquake Research.

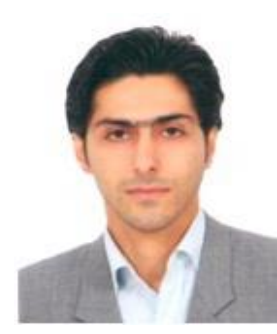

Hamid rajabi was born in Gorgan, Iran. $\mathrm{He}$ received the M.Sc. degree in Power engineering from Azad university of Aliabad.

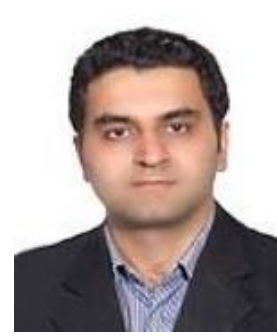

Mohammad Nasrollahnejad was born in Gorgan, Iran. He received the M.Sc. degree in electronic engineering from shahid beheshti university, Tehran. Iran. Now he is PHD student of electronic engineering in semnan university, Iran.

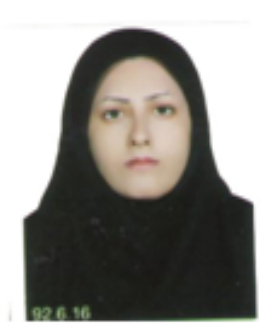

Samaneh Tahmasebi was born in Gorgan, Iran. She is PHD student of mathematics in university of shahrood, Iran. 